\title{
Regularization and Choice of the Parameter for the Third Kind Nonlinear Volterra-Stieltjes Integral Equation Solutions
}

\author{
Nurgul Bedelova1, Avyt Asanov², Zhypar Orozmamatova ${ }^{3}$, Zhypargul Abdullaeva4* ${ }^{*}$ \\ ${ }^{1}$ Department of Mathematics and Information Technologies, Osh State University, Osh, Kyrgyzstan \\ ${ }^{2}$ Department of Mathematics, Kyrgyz-Turkish Manas University, Bishkek, Kyrgyzstan \\ ${ }^{3}$ Department of Economics and Management, Osh Technological University, Osh, Kyrgyzstan \\ ${ }^{4}$ Science and Research Department, Osh State University, Osh, Kyrgyzstan \\ Email: *jypar.science@oshsu.kg
}

How to cite this paper: Bedelova, N., Asanov, A., Orozmamatova, Z. and Abdullaeva, Z. (2021) Regularization and Choice of the Parameter for the Third Kind Nonlinear Volterra-Stieltjes Integral Equation Solutions. International Journal of Modern Nonlinear Theory and Application, 10, 81-90. https://doi.org/10.4236/ijmnta.2021.102006

Received: February 17, 2021

Accepted: May 23, 2021

Published: May 26, 2021

Copyright $\odot 2021$ by author(s) and Scientific Research Publishing Inc. This work is licensed under the Creative Commons Attribution International License (CC BY 4.0). http://creativecommons.org/licenses/by/4.0/

\begin{abstract}
The article is considering the third kind of nonlinear Volterra-Stieltjes integral equations with the solution by Lavrentyev regularizing operator. A uniqueness theorem was proved, and a regularization parameter was chosen. This can be used in further development of the theory of the integral equations in non-standard problems, classes in the numerical solution of third kind Volterra-Stieltjes integral equations, and when solving specific problems that lead to equations of the third kind.
\end{abstract}

\section{Keywords}

Regularization, Solutions, Nonlinear Volterra-Stieltjes Integral Equations, Third Kind, Choice of Regularization Parameter

\section{Introduction}

Differential and integral equations theory considering fractional order are relevant in mathematics nowadays, which have numerous applications in various fields, physics, mechanics, control theory, engineering, electrochemistry, bioengineering, viscoelasticity, porous media [1] [2] [3]. Solution of the nonlinear integral equation of Volterra-Stieltjes type, and the method is based on an equivalence relation between the fractional differential equation, and Volterra-Stieltjes integral equation of the second kind was also reported in our previous works [4] [5]. Here we are describing regularization and the choice of the parameter for nonlinear Volterra-Stieltjes integral equations of the third kind.

Let us consider the equation, 


$$
m(t) v(t)+\int_{t_{0}}^{t} K(t, s, v(s)) \mathrm{d} \varphi(s)=f(t), \quad t \in\left[t_{0}, T\right], \quad T>t_{0}
$$

where $K(t, s, v), f(t), m(t)$ are given functions, $m\left(t_{0}\right)=0, m(t)$ are nondecreasing continuous functions on $\left[t_{0}, T\right], v(t)$ is an unknown function on $\left[t_{0}, T\right], \varphi(t)$ is an increasing continuous function $\left[t_{0}, T\right]$.

Along with Equation (1), we will consider the equation

$$
(\varepsilon+m(t)) v(t, \varepsilon)+\int_{t_{0}}^{t} K(t, s, v(s, \varepsilon)) \mathrm{d} \varphi(s)=f(t)+\varepsilon u\left(t_{0}\right), \quad t \in\left[t_{0}, T\right],
$$

where $0<\varepsilon$ is a small parameter, $(t, s) \in G=\left\{(t, s): t_{0} \leq s \leq t \leq T\right\}$.

Everywhere we assume that $K(t, s, u)$ is representable as

$$
K(t, s, u)=K_{0}(t, s) u+K_{1}(t, s, u),
$$

where

$$
(t, s, u) \in G \times R
$$

Various questions of the theory of the integral equations were investigated in many works. In particular, in [6] linear integral equations of the second kind and their systems on finite and infinite intervals were studied. A survey of results on Volterra integral equations of the second kind was described [7]. The existence of a multiparameter family of solutions proved for linear Volterra integral equations of the first and third kind with smooth kernels [8]. But the fundamental results for the Fredholm integral equations of the first kind were obtained [9], where regularizing operators according to M.M. Lavrentyev were constructed for solving the linear Fredholm integral equations of the first kind. In [10] and [11], Volterra equations of the first kind and inverse problems were investigated. The uniqueness theorems were proved and regularizing operators were constructed according to M.M. Lavrentyev for systems of linear and nonlinear Volterra integral equations of the first kind with nonsmooth matrix kernels [12] [13]. The systems of nonlinear Volterra integral equations of the third kind, uniqueness theorems were proved and regularizing operators were constructed according to M.M. Lavrentyev [14]. In [15], the uniqueness theorems were proved for systems of linear Fredholm integral equations of the third kind, and regularizing operators were constructed according to M.M. Lavrentyev. In [16], based on a new approach, the questions of existence and uniqueness of solutions for systems of linear Fredholm integral equations of the third kind with a singularity at one point on a finite interval were investigated. Based on the approach proposed in [17], the class of Fredholm integral equations of the third kind on a finite interval was studied. Based on the approaches proposed in [18] [19], an improved new approach was developed for studying systems of linear and nonlinear Fredholm integral equations of the third kind with multipoint singularities on a finite interval. In [20], according to the concept of the derivative of a function concerning an increasing function introduced in [19], linear and nonlinear Volterra-Stieltjes integral equations of the first and second kind were investigated. For the solution of one class of linear Volterra, and Volterra-Stieltjes 
integral equations of the third kind, a regularizing operator was constructed according to MM. Lavrentyev and proved the uniqueness theorem [21] [22]. The regularization parameter is chosen for solving the linear Volterra-Stieltjes integral equation of the third kind [4].

Here, to solve the nonlinear Volterra-Stieltjes integral equation of the third kind (1), a regularizing operator which was constructed according to M.M. Lavrentyev, a uniqueness theorem proved, and a regularization parameter was chosen.

Suppose the following conditions are met:

a) $K(t, s) \in C(G), \quad K_{0}(t, t) \in C\left[t_{0}, T\right], \quad K_{0}(t, t) \geq 0$ at $t \in\left[t_{0}, T\right]$

b) If the condition $t>\tau$ for any function $(t, s),(\tau, s) \in G$, the following equation is fair:

$$
\left|K_{0}(t, s)-K_{0}(\tau, s)\right| \leq l_{1}\left[\int_{\tau}^{t} K_{0}(s, s) \mathrm{d} \varphi(s)+m(t)\right],
$$

where $l_{1}$ is a known positive number.

c) $K_{1}(t, t, u)=0,(t, u) \in\left[t_{0}, T\right] \times R, K_{1}(t, s, 0)=0$

at $(t, s) \in G$,

at $t>\tau$ for any $\left(t, s, u_{1}\right),\left(\tau, s, u_{1}\right),\left(t, s, u_{2}\right),\left(\tau, s, u_{2}\right) \in G \times R$ following equation is fair:

$$
\begin{aligned}
& \left|K_{1}\left(t, s, u_{1}\right)-K_{1}\left(\tau, s, u_{1}\right)-K\left(t, s, u_{2}\right)+K\left(\tau, s, u_{2}\right)\right| \\
& \leq I_{2}\left[\int_{\tau}^{t} K_{0}(s, s) \mathrm{d} \varphi(s)+m(t)\right]\left|u_{1}-u_{2}\right|,
\end{aligned}
$$

where $l_{2}$ is a known positive number.

Here $C\left[t_{0}, T\right]$ is the space of all continuous functions $v(t)$, determined on $\left[t_{0}, T\right]$

with norm

$$
\|v(t)\|_{c}=\max _{t \in\left[t_{0}, T\right]}\|v(t)\| .
$$

We will denote $C_{\psi}^{\gamma}\left[t_{0}, T\right], 0<\gamma \leq 1$ linear space of all functions $v(t)$, determined on $\left[t_{0}, T\right]$ and satisfying condition

$$
|v(t)-v(s)| \leq M|\psi(t)-\psi(s)|^{\gamma}, \quad \psi(t)=\int_{t_{0}}^{t} K_{0}(s, s) \mathrm{d} \varphi(s)+m(t)
$$

where $M$ is a positive constant depending on $v(t)$, but not on the $t$ and $s$.

In further the lemmas 1,2 and 3 are used,

Lemma 1.

Let conditions a) holds and

$$
\begin{aligned}
F(t, \varepsilon)= & -\frac{\varepsilon v(t)}{\varepsilon+m(t)} \mathrm{e}^{-\int^{t} \frac{K(\tau, \tau)}{\varepsilon+m(\tau)} \mathrm{d} \varphi(\tau)} \\
& -\int_{t_{0}}^{t} \frac{K_{0}(s, s)}{\varepsilon+m(t)} \mathrm{e}^{-\int^{t} \frac{K_{0}(\tau, \tau)}{\varepsilon+m(\tau)} \mathrm{d} \varphi(\tau)} \frac{\varepsilon[v(t)-v(s)]}{\varepsilon+m(s)} \mathrm{d} \varphi(s) .
\end{aligned}
$$




$$
\text { If } \begin{aligned}
v(t) \in C_{\psi}^{\gamma}\left[t_{0}, T\right], 0<\gamma<1, v\left(t_{0}\right)=0, \text { then } \\
\qquad F(t, \varepsilon) \|_{c} \leq M\left(M_{1}+M_{2}\right) \varepsilon^{\gamma},
\end{aligned}
$$

where

$$
\begin{gathered}
M=\sup _{t, s \in\left[t_{0}, T\right]}|v(t)-v(s)| /|\psi(t)-\psi(s)|^{\gamma} \\
M_{1}=\sup _{\mu \geq 0}\left[\mu^{\gamma} \mathrm{e}^{-\mu}\right], M_{2}=\int_{0}^{\infty} \mathrm{e}^{-z} z^{\gamma} \mathrm{d} z .
\end{gathered}
$$

\section{Lemma 2.}

Let conditions a), b) hold and

$$
\begin{aligned}
H_{0}(t, \tau, \varepsilon)= & -\frac{1}{\varepsilon+m(t)}\left[K_{0}(t, \tau)-K_{0}(\tau, \tau)\right] \\
& +\int_{\tau}^{t} \frac{K_{0}(s, s)}{\varepsilon+m(t)} \mathrm{e}^{-\int \frac{K_{0}(\tau, \tau)}{\varepsilon+m(\tau)} \mathrm{d} \varphi(\tau)} \frac{1}{\varepsilon+m(s)}\left[K_{0}(s, \tau)-K_{0}(\tau, \tau)\right] \mathrm{d} \varphi(s) .
\end{aligned}
$$

The following estimate is fair

$$
\left|H_{0}(t, \tau, \varepsilon)\right| \leq(e+1) l_{1}, \quad(t, \tau) \in G, \varepsilon>0 .
$$

\section{Lemma 3.}

Let conditions a), c) hold and

$$
\begin{aligned}
H(t, \tau, \xi(\tau, \varepsilon))= & -\frac{1}{\varepsilon+m(t)}\left[K_{1}(t, \tau, u(\tau)+\xi(\tau, \varepsilon))-K_{1}(t, \tau, u(\tau))\right] \\
& +\int_{\tau}^{t} \frac{K_{0}(s, s)}{\varepsilon+m(s)} \mathrm{e}^{-\int^{t} \frac{K_{0}(\tau, \tau)}{\varepsilon+m(\tau)} \mathrm{d} \varphi(\tau)} \frac{1}{\varepsilon+m(t)}\left[K_{1}(s, \tau, u(\tau)+\xi(\tau, \varepsilon))(7)\right. \\
& \left.-K_{1}(s, \tau, u(\tau))\right] \mathrm{d} \varphi(\tau) .
\end{aligned}
$$

If that, the following estimation is fair

$$
|H(t, \tau, \xi(\tau, \varepsilon))| \leq l_{2}(1+e) \xi(\tau, \varepsilon), \quad(t, \tau, \varepsilon) \in G \times R, \quad \varepsilon>0
$$

\section{Theorem 1.}

Let the conditions a), b), c) be satisfied, and Equation (1) has a solution

$$
u(t) \in C_{\psi}^{\gamma}\left[t_{0}, T\right], \quad 0<\gamma \leq 1 .
$$

Then solution $v(t, \varepsilon)$ of the Equation (2) converges in the norm $C\left[t_{0}, T\right]$ to $u(t)$ for $\varepsilon \rightarrow 0$ and the estimate

$$
\|v(t, \varepsilon)-u(t)\|_{c} \leq K M M_{3} \varepsilon^{\gamma},
$$

holds. Where

$$
\begin{gathered}
M=\sup _{t, s \in\left[t_{0}, T\right]} \frac{|u(t)-u(s)|}{|\psi(t)-\psi(s)|^{\gamma}}, M_{1}=\sup _{v \geq 0}\left(v^{\gamma} \mathrm{e}^{-v}\right), \\
M_{2}=\int_{0}^{\infty} \mathrm{e}^{-z} z^{\gamma} \mathrm{d} z, M_{3}=\left(M_{1}+M_{2}\right) e, \\
K=\exp \left\{(1+e)\left(l_{1}+l_{2}\right)\left[\varphi(T)-\varphi\left(t_{0}\right)\right]\right\} .
\end{gathered}
$$


Further let us consider that function $f_{\delta}(t) \in C\left[t_{0}, T\right]$ and number $u_{0}$, in agreement with

$$
\left\|f(t)-f_{\delta}(t)\right\|_{c} \leq \delta, \quad\left|u\left(t_{0}\right)-u_{0}\right| \leq \alpha \delta,
$$

where $0<\alpha$ and $0<\delta$ are constant values.

Let us consider the equation

$$
(\varepsilon+m(t)) v_{\delta}(t, \varepsilon)+\int_{t_{0}}^{t} K\left(t, s, v_{\delta}(s, \varepsilon)\right) \mathrm{d} \varphi(s)=f_{\delta}(t)+\varepsilon u_{0}, \quad t \in\left[t_{0}, T\right]
$$

From (2) by subtracting formula (11) and introducing the notation

$$
u_{\delta}(t, \varepsilon)=v(t, \varepsilon)-v_{\delta}(t, \varepsilon), \quad t \in\left[t_{0}, T\right] .
$$

We have

$$
\begin{aligned}
& (\varepsilon+m(t)) u_{\delta}(t, \varepsilon)+\int_{t_{0}}^{t} K_{0}(t, s) u_{\delta}(s, \varepsilon) \mathrm{d} \varphi(s) \\
& +\int_{t_{0}}^{t}\left[K_{1}(t, s, v(s, \varepsilon))-K_{1}\left(t, s, u_{\delta}(s, \varepsilon)\right)\right] \mathrm{d} \varphi(s) \\
& =f(t)-f_{\delta}(t)+\varepsilon\left(u\left(t_{0}\right)-u_{0}\right), \quad t \in\left[t_{0}, T\right] .
\end{aligned}
$$

Equation (13) can be written in the form

$$
\begin{aligned}
& u_{\delta}(t, \varepsilon)+\int_{t_{0}}^{t} \frac{K_{0}(s, s)}{\varepsilon+m(t)} u_{\delta}(s, \varepsilon) \mathrm{d} \varphi(s) \\
& +\int_{t_{0}}^{t} \frac{1}{\varepsilon+m(t)}\left[K_{0}(t, s)-K_{0}(s, s)\right] u_{\delta}(s, \varepsilon) \mathrm{d} \varphi(s) \\
& +\int_{t_{0}}^{t} \frac{1}{\varepsilon+m(t)}\left[K_{1}(t, s, v(s, \varepsilon))-K_{1}\left(t, s, u_{\delta}(s, \varepsilon)\right)\right] \mathrm{d} \varphi(s) \\
& =\frac{f(t)-f_{\delta}(t)}{\varepsilon+m(t)}+\frac{\varepsilon\left[u\left(t_{0}\right)-u_{0}\right]}{\varepsilon+m(t)}, t \in\left[t_{0}, T\right] .
\end{aligned}
$$

Using the kernel resolvents $\left[-\frac{K_{0}(s, s)}{\varepsilon+m(t)}\right]$, and generalized Dirichlet formula [15], Equation (14) is reduced to the following equivalent equation

$$
\begin{aligned}
u_{\delta}(t, \varepsilon)= & \int_{t_{0}}^{t} H_{0}(t, s, \varepsilon) u_{\delta}(s, \varepsilon) \mathrm{d} \varphi(s) \\
& +\int_{t_{0}}^{t} P\left(t, \tau, v(\tau, \varepsilon), v_{\delta}(\tau, \varepsilon)\right) \mathrm{d} \varphi(\tau)+F_{\delta}(t, \varepsilon)
\end{aligned}
$$

where $H_{0}(t, s, \varepsilon)$ was determined in the lemma 2,

$$
\begin{aligned}
F_{\delta}(t, \varepsilon)= & \frac{f(t)-f_{\delta}(t)}{\varepsilon+m(t)}+\frac{\varepsilon\left[u\left(t_{0}\right)-u_{0}\right]}{\varepsilon+m(t)}-\frac{1}{\varepsilon+m(t)} \int_{t_{0}}^{t} K_{0}(s, s) \mathrm{e}^{-\int \frac{t}{s} \frac{K_{0}(\tau, \tau)}{\varepsilon+m(\tau)} \mathrm{d} \varphi(\tau)} \\
& *\left[\frac{f(s)-f_{\delta}(s)}{\varepsilon+m(s)}+\frac{\varepsilon\left[u\left(t_{0}\right)-u_{0}\right]}{\varepsilon+m(s)}\right] \mathrm{d} \varphi(s),
\end{aligned}
$$




$$
\begin{aligned}
& P\left(t, \tau, v(\tau, \varepsilon), v_{\delta}(\tau, \varepsilon)\right) \\
& =\frac{-1}{\varepsilon+m(t)}\left[K_{1}(t, \tau, v(\tau, \varepsilon))-K_{1}\left(t, \tau, v_{\delta}(\tau, \varepsilon)\right)\right] \\
& +\int_{\tau}^{t} \frac{K_{0}(s, s)}{\varepsilon+m(t)} e^{-\int \frac{\int_{0}(\tau, \tau)}{\varepsilon+m(\tau)} \mathrm{d} \varphi(\tau)} \frac{1}{\varepsilon+m(s)}\left[K_{1}(s, \tau, v(\tau, \varepsilon))\right. \\
& \left.-K_{1}\left(s, \tau, u_{\delta}(\tau, \varepsilon)\right)\right] \mathrm{d} \varphi(s) .
\end{aligned}
$$

It is not hard to be convinced that

$$
\begin{aligned}
& \frac{-1}{\varepsilon+m(t)}\left[K_{1}(t, \tau, v(\tau, \varepsilon))-K_{1}\left(t, \tau, v_{\delta}(\tau, \varepsilon)\right)\right] \\
& =\frac{-1}{\varepsilon+m(t)} \mathrm{e}^{-\int \frac{t}{\tau} K_{0}(\tau, \tau)} \frac{1+\tau(\tau)}{\varepsilon+(\tau)}\left[K_{1}(t, \tau, v(\tau, \varepsilon))-K_{1}\left(t, \tau, v_{\delta}(\tau, \varepsilon)\right)\right] \\
& -\int_{\tau}^{t} \frac{K_{0}(s, s)}{(\varepsilon+m(t))(\varepsilon+m(s))} \mathrm{e}^{-\int \frac{\int_{0}(\tau, \tau)}{\varepsilon+m(\tau)} d \varphi(\tau)}\left[K_{1}(t, \tau, v(\tau, \varepsilon))\right. \\
& \left.-K_{1}\left(t, \tau, v_{\delta}(\tau, \varepsilon)\right)\right] \mathrm{d} \varphi(s) .
\end{aligned}
$$

Taking into account condition c) and identity (18), from (17) we have

$$
\begin{aligned}
& P\left(t, \tau, v(\tau, \varepsilon), v_{\delta}(\tau, \varepsilon)\right) \\
= & \frac{-1}{\varepsilon+m(t)} \mathrm{e}^{-\int \frac{K_{0}(\tau, \tau)}{\varepsilon+m(\tau)} \mathrm{d} \varphi(\tau)}\left[K_{1}(t, \tau, v(\tau, \varepsilon))-K(\tau, \tau, v(\tau, \varepsilon))\right. \\
& \left.+K\left(\tau, \tau, v_{\delta}(\tau, \varepsilon)\right)-K_{1}\left(t, \tau, v_{\delta}(\tau, \varepsilon)\right)\right] \\
& -\int_{\tau}^{t} \frac{K_{0}(s, s)}{(\varepsilon+m(t))(\varepsilon+m(s))} \mathrm{e}^{-\int^{t} \frac{K_{0}(\tau, \tau)}{s+m(\tau)} \mathrm{d} \varphi(\tau)}\left[K_{1}(t, \tau, v(\tau, \varepsilon))\right. \\
& \left.-K_{1}\left(t, \tau, v_{\delta}(\tau, \varepsilon)\right)-K_{1}(s, \tau, v(\tau, \varepsilon))+K_{1}\left(s, \tau, v_{\delta}(\tau, \varepsilon)\right)\right] \mathrm{d} \varphi(s) .
\end{aligned}
$$

Based on the Equation (10), from (16) we have

$$
\left\|F_{\delta}(t, \varepsilon)\right\|_{c} \leq 2\left(\frac{\delta}{\varepsilon}+\alpha \delta\right)
$$

Based on Lemma 2, $H_{0}(t, s, \varepsilon)$ estimate (6) is fair.

By estimating $P\left(t, \tau, v(\tau, \varepsilon), u_{0}(\tau, \varepsilon)\right)$. Taking into account conditions a) and c) from (19) we obtain

$$
\begin{aligned}
& \left|P\left(t, \tau, v(\tau, \varepsilon), v_{\delta}(\tau, \varepsilon)\right)\right| \\
& \leq \frac{l_{2}}{\varepsilon+m(t)} \mathrm{e}^{-\int \frac{K_{0}(\tau, \tau)}{\tau+m(\tau)} \mathrm{d} \varphi(\tau)}\left[\int_{\tau}^{t} K_{0}(\tau, \tau) \mathrm{d} \varphi(\tau)+m(t)\right]\left|v(\tau, \varepsilon)-v_{\delta}(\tau, \varepsilon)\right| \\
& \quad+\int_{\tau}^{t} \frac{K_{0}(s, s) l_{2}}{(\varepsilon+m(t))(\varepsilon+m(s))}\left[\int_{s}^{t} K_{0}(\tau, \tau) \mathrm{d} \varphi(\tau)+m(t)\right] \\
& \quad \times \mathrm{e}^{-\int^{t} \frac{K_{0}(\tau, \tau)}{\varepsilon+m(\tau)} \mathrm{d} \varphi(\tau)}\left|v(\tau, \varepsilon)-v_{\delta}(\tau, \varepsilon)\right| \mathrm{d} \varphi(s)
\end{aligned}
$$




$$
\begin{aligned}
& \leq I_{2}\left|v(\tau, \varepsilon)-v_{\delta}(\tau, \varepsilon)\right|\left\{e^{-\left[\frac{t}{\tau} \frac{K_{0}(s, s)}{\varepsilon+m(s)} \mathrm{d} \varphi(s)+\frac{m(t)}{\varepsilon+m(t)}\right]}\left[\int_{\tau}^{t} \frac{K_{0}(s, s)}{\varepsilon+m(s)} \mathrm{d} \varphi(s)+\frac{m(t)}{\varepsilon+m(t)}\right]\right. \\
& \left.+\int_{\tau}^{t} \frac{K_{0}(s, s) e}{\varepsilon+m(s)} \mathrm{e}^{-\left[\int_{s}^{t} \frac{K_{0}(\tau, \tau)}{\varepsilon+m(\tau)} \mathrm{d} \varphi(\tau)+\frac{m(t)}{\varepsilon+m(t)}\right]}\left[\int_{s}^{t} \frac{K_{0}(\tau, \tau)}{\varepsilon+m(\tau)} \mathrm{d} \varphi(\tau)+\frac{m(t)}{\varepsilon+m(t)}\right] \mathrm{d} \varphi(s)\right\} \\
& \leq l_{2} e\left[\sup _{v \geq 0}\left(\mathrm{e}^{-v} v\right)+\int_{0}^{\infty} \mathrm{e}^{-v} v \mathrm{~d} v\right]\left|v(\tau, \varepsilon)-v_{\delta}(\tau, \varepsilon)\right| \\
& =l_{2}(e+1)\left|v(\tau, \varepsilon)-v_{\delta}(\tau, \varepsilon)\right| \text {. }
\end{aligned}
$$

Based on the estimate (20), (6), (21) and taking into account (12), from (15) we have

$$
\left|u_{\delta}(t, \varepsilon)\right| \leq \int_{t_{0}}^{t}\left(l_{1}+l_{2}\right)(e+1)\left|u_{\delta}(s, \varepsilon)\right| \mathrm{d} \varphi(s)+2\left(\frac{\delta}{\varepsilon}+\alpha \delta\right), \quad t \in\left[t_{0}, T\right] .
$$

Further, based on the generalized Gronwall-Bellman inequality [6], from (22) we obtain the following estimate

$$
\left\|u_{\delta}(t, \varepsilon)\right\|_{c} \leq M_{4}\left(\frac{\delta}{\varepsilon}+\alpha \delta\right)
$$

where

$$
M_{4}=2 \exp \left\{\left(l_{1}+l_{2}\right)(e+1)\left[\varphi(T)-\varphi\left(t_{0}\right)\right]\right\} .
$$

It is known that

$$
\left\|v_{\delta}(t, \varepsilon)-v(t)\right\|_{c} \leq\left\|u_{\delta}(t, \varepsilon)\right\|_{c}+\|v(t, \varepsilon)-v(t)\|_{c} .
$$

Here taking into account (23), we have

$$
\left\|v_{\delta}(t, \varepsilon)-v(t)\right\|_{c} \leq M_{4}\left(\frac{\delta}{\varepsilon}+\alpha \delta\right)+\|v(t, \varepsilon)-v(t)\|_{c} .
$$

where number $M_{4}$ determined by the formula (24).

$$
\left\|v_{\delta}(t, \varepsilon)-v(t)\right\|_{c} \leq M_{4}\left(\frac{\delta}{\varepsilon}+\alpha \delta\right)+M_{5},
$$

where $M_{5}=K M M_{3}$, numbers $K, M$ and $M_{3}$ were determined in Theorem 1 . Assuming $\varepsilon=\delta^{\frac{1}{2}}$ from (26) we obtain

$$
\left\|v_{\delta}\left(t, \delta^{\frac{1}{2}}\right)-u(t)\right\|_{c} \leq M_{4}\left(\delta^{\frac{1}{2}}+\alpha \delta\right)+M_{5} \delta^{\frac{\gamma}{2}} .
$$

where numbers $M_{4}, M_{5}$ are determined in Equations (24) and (26).

Thus, Theorem 2 was proved.

Theorem 2. Let conditions a), b), c) be satisfied, and Equation (1) has a solution

$$
v(t) \in C_{\psi}^{\gamma}\left[t_{0}, T\right], \quad 0<\gamma \leq 1
$$




$$
\psi(t)=\int_{t_{0}}^{t} K(s, s) \mathrm{d} \varphi(s)+m(t), \quad t \in\left[t_{0}, T\right] .
$$

Then the solution $v_{\delta}(t, \varepsilon)$ in Equation (11) $\varepsilon=\delta^{\frac{1}{2}} \rightarrow 0$ converges at the norm

$$
C\left[t_{0}, T\right] \text { to } v(t) \text {. }
$$

Wherein, Estimate (27) is fair.

Example. Let us consider Equations (1) at

$$
\begin{aligned}
& t_{0}=0, T=1, \varphi(t)=\sqrt{t}, K_{0}(t, s)=(1+t)(1-\sqrt{s}), \\
& m(t)=t, K_{1}(t, s, v)=(t-s) \frac{v}{1+v^{2}}, t \in[0,1],
\end{aligned}
$$

i.e., let us look at the following equation

$$
t v(t)+\int_{0}^{t}\left[(1+t)(1-\sqrt{s}) v(s)+\frac{(t-s) v(s)}{1+v^{2}(s)}\right] \mathrm{d}(\sqrt{s})=f(t), \quad t \in[0,1] .
$$

In this case, conditions a), b), c) of Theorems 1 and 2 are satisfied. Since at conditions $t>\eta, t, \eta \in[0,1]$, the following estimate is fair

$$
\left|K_{0}(t, s)-K_{0}(\eta, s)\right|=(t-\eta)(1-\sqrt{s}) \leq m(t) \leq \int_{\eta}^{t} K_{0}(s, s) \mathrm{d} \varphi(s)+m(t) .
$$

Here $l_{1}=1$.

At $t>\tau$ for $\left(t, s, u_{1}\right),\left(t, s, u_{2}\right),\left(\tau, s, u_{1}\right),\left(\tau, s, u_{2}\right) \in G \times R$

the following estimate is fair

$$
\begin{aligned}
& \left|K_{1}\left(t, s, u_{1}\right)-K_{1}\left(\tau, s, u_{1}\right)-K_{1}\left(t, s, u_{2}\right)+K_{1}\left(\tau, s, u_{2}\right)\right| \\
& \leq(t-\tau)\left|\frac{v_{1}}{1+v_{1}^{2}}-\frac{v_{2}}{1+v_{2}^{2}}\right| \\
& \leq(t-\tau)\left|v_{1}-v_{2}\right| \frac{1+\left|v_{1}\right|\left|v_{2}\right|}{\left(1+v_{1}^{2}\right)\left(1+v_{2}^{2}\right)} \\
& \leq(t-\tau)\left|v_{1}-v_{2}\right|, \text { In this way } l_{2}=1 .
\end{aligned}
$$

\section{Conclusions}

After choosing the regularization parameter for solving nonlinear Volterra-Stieltjes integral equations of the third kind, we made the following conclusions:

1) Sufficient uniqueness conditions and regularization of solutions of nonlinear Volterra-Stieltjes integral equations of the third kind were found;

2) The choice of the regularization parameter for solving a class of VolterraStieltjes nonlinear equations of the third kind was considered;

3 Uniqueness theorems for solutions proved for the nonlinear Volterra-Stieltjes integral equations of the third kind.

\section{Acknowledgements}

The authors are thankful to Professor A. Asanov for discussions and advice in 
solving equations.

\section{Conflicts of Interest}

The authors declare no conflicts of interest regarding the publication of this paper.

\section{References}

[1] Asanov, A., Hazar, E., Eroz, M., Matanova, K. and Abdyldaeva, E. (2016) Approximate Solution of Volterra-Stieltjes Linear Integral Equations of the Second Kind with the Generalized Trapezoid Rule. Advances in Mathematical Physics, 2016, Article ID: 1798050. https://doi.org/10.1155/2016/1798050

[2] Ezzati, R., Salahshour, S., Yager, R.R. and Khodabin, M. (2014) Fuzzy Linear and Nonlinear Integral Equations: Numerical Methods. Abstract and Applied Analysis, 2014, Article ID: 147351 . https://doi.org/10.1155/2014/147351

[3] Gordji, M.E., Baghani, H. and Baghani, O. (2011) On Existence and Uniqueness of Solutions of a Nonlinear Integral Equation. Journal of Applied Mathematics, 2011, Article ID: 743923. https://doi.org/10.1155/2011/743923

[4] Bedelova, N. (2020) The Choice of the Regularization Parameter for Solving Linear Volterra-Stieltjes Integral Equations of the Third Kind. In: Popkova, E. and Sergi, B., Eds., Scientific and Technical Revolution: Yesterday, Today and Tomorrow, ISC 2019, Lecture Notes in Networks and Systems, Vol. 129, Springer, Cham, 321-328. https://doi.org/10.1007/978-3-030-47945-9_36

[5] Asanov, A., Almeida, R. and Malinowska, A.B. (2019) Fractional Differential Equations and Volterra-Stieltjes Integral Equations of the Second Kind. Computational and Applied Mathematics, 38, Article No. 160. https://doi.org/10.1007/s40314-019-0941-2

[6] Mikhlin, S.G. (1959) Lectures on Linear Integral Equations. Fizmatgiz, Moscow, 234 p.

[7] Tsalyuk, Z.B. (1977) Volterra Integral Equations. Itogi Nauki i Tekhniki. Ser. Mat. Analysis. Moscow, 15, 131-198.

[8] Lavrentyev, M.M. (1959) Integral Equations of the First Kind. Dokl. USSR Academy of Sciences, 127, 31-33.

[9] Bughgeim, A.L. (1999) Volterra Equations and Inverse Problems. VSP, Utrecht, 204 p. https://doi.org/10.1515/9783110943245

[10] Denisov, A.M. (1999) Elements to the Theory of Inverse Problems. VSP, Utrecht, 272 p. https://doi.org/10.1515/9783110943252

[11] Asanov, A. (1998) Regularization, Uniqueness and Existence of Solutions of Volterra Equations of the First Kind. VSP, Utrecht, 276 p. https://doi.org/10.1515/9783110943238

[12] Imanaliev, M.I. and Asanov, A. (1989) On Solutions of Systems of Volterra Nonlinear Integral Equations of the first KIND. Dokl. USSR Academy of Sciences, 309, 1052-1055.

[13] Imanaliev, M.I., and Asanov, A. (2007) Regularization and Uniqueness of Solutions of Systems of Nonlinear Volterra Integral Equations of the Third Kind. Doklady Mathematics, 76, 490-493. https://doi.org/10.1134/S1064562407040035

[14] Imanaliev, M.I. and Asanov, A. (2010) Solutions to Systems of Linear Fredholm Integral Equations of the Third Kind. Doklady Mathematics, 81, 115-118. 
https://doi.org/10.1134/S1064562410010321

[15] Imanaliev, M.I., Asanov, A. and Asanov, R.A. (2011) A Class of Systems of Linear Fredholm Integral Equations of the Third Kind. Doklady Mathematics, 83, 227-231.

[16] Asanov, A., Matanova, K.B. and Asanov, R.A. (2017) A Class of Linear and Nonlinear Fredholm Integral Equations of the Third Kind. Kuwait Journal of Science, 44, 17-28.

[17] Imanaliev, M.I., Asanov, A. and Asanov, R.A. (2017) Solutions to Systems of Linear Fredholm Integral Equations of the Third Kind with Multipoint Singularities. Doklady Mathematics, 95, 235-239.

[18] Imanaliev, M.I., Asanov, A. and Asanov, R.A. (2018) On a Class of Systems of Linear and Nonlinear Fredholm Integral Equations of the Third Kind with Multipoint Singularities. Differential Equations, 54, 381-391.

https://doi.org/10.1134/S0012266118030096

[19] Asanov, A. (2001) Derivative Function with Respect to Increasing Function. Journal of Natural Sciences KTUM, 1, 18-64.

[20] Asanov, A. (2002) Volterra-Stieltjes Integral Equations of the Second and First Kind. Journal of Natural Sciences, KTUM, 2, 79-95.

[21] Asanov, A. and Bedelova, N.S. (2014) One Class of Linear Integral Volterra-Stieltjes Equations of the Third Kind. Bulletin of KazNPU Named after Abay, Almaty, 4, 8-13.

[22] Liu, Z., Lee, S. and Kang, S.M. (2012) Solvability of Nonlinear Integral Equations of Volterra Type. Abstract and Applied Analysis, 2012, Article ID: 932019.

https://doi.org/10.1155/2012/932019 